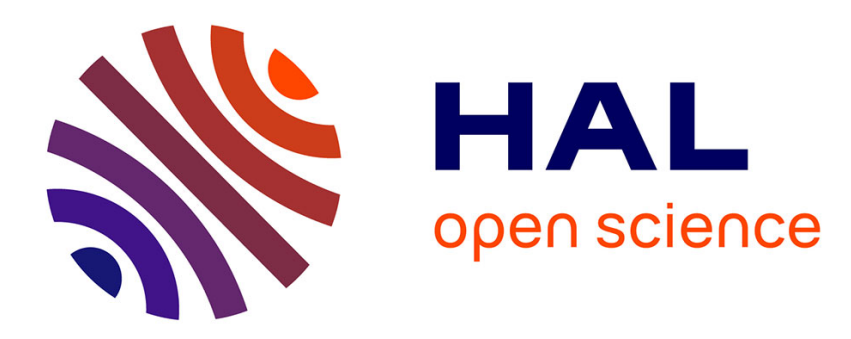

\title{
Water waves as a spatial dynamical system; infinite depth case
}

\author{
Gérard Iooss, Matthieu Barrandon
}

\section{To cite this version:}

Gérard Iooss, Matthieu Barrandon. Water waves as a spatial dynamical system; infinite depth case. Chaos: An Interdisciplinary Journal of Nonlinear Science, 2005, 15, pp.037112. 10.1063/1.1940387 . hal-00012888

\section{HAL Id: hal-00012888 \\ https://hal.science/hal-00012888}

Submitted on 28 Oct 2005

HAL is a multi-disciplinary open access archive for the deposit and dissemination of scientific research documents, whether they are published or not. The documents may come from teaching and research institutions in France or abroad, or from public or private research centers.
L'archive ouverte pluridisciplinaire HAL, est destinée au dépôt et à la diffusion de documents scientifiques de niveau recherche, publiés ou non, émanant des établissements d'enseignement et de recherche français ou étrangers, des laboratoires publics ou privés. 


\title{
Water waves as a spatial dynamical system; infinite depth case
}

\author{
Matthieu Barrandon, Gérard Iooss \\ INLN, UMR CNRS-UNSA 6618 \\ 1361 route des Lucioles, 06560 Valbonne, France
}

\begin{abstract}
We review the mathematical results on travelling waves in one or several superposed layers of potential flow, subject to gravity, with or without surface and interfacial tension, where the bottom layer in infinitely deep. The problem is formulated as a "spatial dynamical system" and it is shown that the linearized operator of the resulting reversible system, has an essential spectrum filling the real line. We consider 3 examples where bifurcation occurs. i) The first example is when in moving a parameter, two pairs of imaginary eigenvalues merge into one pair of double eigenvalues, and then split into 4 symmetric complex conjugate eigenvalues. ii) The second example is when one pair of imaginary eigenvalues meet in 0 , and disappear; iii) the third example is when the phenomenon described at ii) is superposed to the presence of another pair of imaginary eigenvalues sitting at finite distance from 0 . We give a physical example for each case and more specially study the solitary waves and generalized solitary waves, emphasizing the differences, in the methods and in the results, between these cases and the finite depth case.
\end{abstract}

Keywords: reversible bifurcations theory, nonlinear water waves, travelling waves, solitary waves, infinitedimensional reversible dynamical systems, essential spectrum.

The mathematical study of travelling waves, in the context of two dimensional potential flows in one or several layers of perfect fluid(s), in the presence of free surface and interfaces can be set as an ill-posed evolution problem, where the horizontal space variable plays the role of a "time".

A case of great physical interest is the infinite depth limit. In such a case, the classical reduction technique to a small-dimensional center manifold fails because the linearized operator possesses an essential spectrum filling the whole real axis, and adapted tools are necessary. We give a method and the results for different types of systems. A first example is with a single infinitely deep layer, with surface tension at the free surface, where the bifurcation occurs when two pairs of imaginary eigenvalues meet and split into 2 pairs of complex eigenvalues. This case leads to solitary waves with polynomially (instead of exponentially) damped oscillations at infinity [1]. Another example is with two superposed layers, the bottom one being infinitely deep, with no surface tension at the interface. In case of a strong enough surface tension at the free surface, the bifurcation occurs when a pair of imaginary eigenvalues merge at 0 , which is part of the essential spectrum, and disappear when a parameter is varying. In case of no surface tension at the free surface, there is in addition an oscillating mode. In both cases the bifurcating solutions are ruled at main order by the Benjamin-Ono nonlocal differential equation, coupled, in the latter case with an oscillatory mode. The first case leads to a one-parameter family of solitary waves [2], and a two-parameters family of periodic waves [3], forming a phase portrait analogous to the one for the corresponding 3-dimensional reversible bifurcation case, except the asymptotics at infinity which is now polyno- mial for the solitary waves. In the second case this spatial dynamics is coupled with a nonlinear oscillator, and leads to the bifurcation of a family of generalized solitary waves, tending at infinity towards periodic waves [4]. The amplitude of these limiting periodic waves cannot vanish in general, and their minimal size is exponentially small [5].

\section{INTRODUCTION}

The search for travelling waves in a system of superposed perfect fluid layers, having a potential flow in each layer, and being subjected to gravity, with possibly surface tension at the free surface and interfaces, may be formulated as a "spatial dynamical system". Such "spatial dynamics" was introduced in the 80's by K. Kirchgässner [6]. Writing the system in the frame moving with the velocity of the travelling wave, we look for steady solutions. Then choosing the spatial coordinate $x$ as a "time" coordinate, the initial value problem in $x$ is ill-posed, but since we are looking for solutions bounded on all the real line, this leads to a sort of "boundary value" problem. Due to Galilean invariance of the physical system, our problem have still a reflection symmetry $x \rightarrow-x$, which leads to a reversible dynamical system meaning that the vector field anticommutes with a symmetry $S$. An easy consequence is that if $U(x)$ is a solution then $S U(-x)$ is also a solution. The spatial dynamics consideration allows in particular to study the asymptotics at infinity. For example, a periodic solution or a homoclinic orbit correspond respectively to periodic travelling waves or to a solitary wave. A review of results concerning problems where all layers have a finite thickness and treated as a spatial dynamical system, is made in the paper [7]. In the present paper we review the results where one layer is infinitely deep. 
We show that the system may be formulated as

$$
\frac{d U}{d x}=L_{\varepsilon} U+N_{\varepsilon}(U)
$$

where $U(x)$ lies in a function space and $U=0$ is an equilibrium solution for all parameter values $\varepsilon \in \mathbb{R}$ corresponding to the rest state for all fluid layers, with a flat free surface and flat interfaces. The linear operator $L_{\varepsilon}$ depends on $\varepsilon$, and the nonlinear operator $N_{\varepsilon}$ is at least quadratic in $U$. The operator $L_{\varepsilon}$ has an essential spectrum filling the entire real line, being the limit of a set of accumulating eigenvalues on the real axis. In addition of this essential spectrum, there are isolated eigenvalues of finite multiplicities given by the dispersion equation.

The first example consists in a single infinitely deep layer, with surface tension at the free surface, where the bifurcation occurs when two pairs of imaginary eigenvalues meet and split into 2 pairs of complex eigenvalues (1:1 reversible resonance). This case leads to solitary waves with polynomially (instead of exponentially) damped oscillations at infinity [1]. Another example is with two superposed layers, the bottom one being infinitely deep, with no surface tension at the interface and strong enough surface tension at the free surface. The bifurcation occurs when a pair of imaginary eigenvalues merge at 0 , which is part of the essential spectrum, and disappears when the parameter is varied. In case of no surface tension at the free surface, there is in addition a pair of simple imaginary eigenvalues. In both cases the bifurcating solutions are ruled at main order by the Benjamin-Ono nonlocal differential equation, coupled, in the latter case with an oscillatory mode. The first case leads to a one-parameter family of solitary waves [2], and a two-parameters family of periodic waves [3] forming a phase portrait analogous to the one of the 3-dimensional reversible bifurcation case, except the asymptotics at infinity which is now polynomial for the solitary waves. In the second case this spatial dynamics is coupled with a nonlinear oscillator, and leads to the bifurcation of a family of periodic travelling waves [8] with in addition two families of generalized symmetric solitary waves, tending at infinity towards periodic waves [4]. The amplitude of these limiting periodic waves is non zero in general, but their minimal size is exponentially small [5].

It should be noticed that local results ( $U$ near 0 ) rely in general on the structure of the spectrum of the linear operator $L_{\varepsilon}$. In the case where all layers have a finite thickness, the spectrum is discrete, and it is possible to use the method of reduction to a small-dimensional center manifold, and then be able to study all small bounded solutions of a reversible differential equation, which is integrable at lowest orders (see a review of the results using such a reduction method in [7]). For applying this method, the linear operator $L_{\varepsilon}$ must have a spectral gap near the imaginary axis. In such a case the description of the finite spectrum near the imaginary axis is sufficient to understand the dynamics of small reversible solutions of (1).
However, many physical situations are such that for obtaining a quantitatively significant result, we have interest to consider the bottom layer as infinitely deep. This is often related with the ratio between the depth and the capillary length scale. This ratio is large in general, which implies that a set of eigenvalues of $L_{\varepsilon}$ are accumulating on the whole real line, hence in particular near 0 . This prevents to obtain a significant domain of validity for the parameter range of values, since the distance of critical eigenvalues (the ones provocating bifurcation) should be small with respect to remaining eigenvalues for applying center manifold reduction. When the depth becomes infinite, the real line lies entirely in the spectrum of $L_{\varepsilon}$ (with no real eigenvalue except 0 ). Since the entire real axis belongs to the spectrum, there is no spectral gap in our problem and we cannot use a center manifold reduction theorem. The absence of spectral gap does not add a big problem when one looks for periodic solutions, since quite simple properties of the nonlinear term still lead to the same result as in finite dimensions (in this later case, given by Lyapunov - Devaney theorem) (see [8] for typical results for travelling water waves). The object of this paper is to review results in such cases for other types of solutions.

We shall observe that most qualitative results obtained in the finite depth case remain valid for the infinite depth case, except that the asymptotic limits at infinity are polynomial instead of exponential. The method strongly relies on a precise knowledge of the resolvent operator $\left(i k-L_{\varepsilon}\right)^{-1}$ near $k=0$, for $k$ real. The Fourier transform of (1) is used for catching solutions tending towards 0 at infinity, while Fourier expansion of (1) is used for the search of periodic solutions, and an adaptation of these tools is used for generalized solitary waves. In the two last examples, 0 is an eigenvalue of $L_{\varepsilon}$, which corresponds to the existence of a family of flows where the upper layer slides with a constant velocity over the bottom one. The corresponding eigenvector is used for the splitting of the system into a scalar equation for some real amplitude coupled with a complementary part which is shown to be a nonlocal function of this real amplitude. When there are oscillating modes, we use them also for the splitting of the system, and the complementary part is shown to be a nonlocal function as well of the corresponding complex amplitude of the oscillating mode, as of the amplitude of the 0 mode. In the first example, the 0 -mode does not exist, and it remains a fouth order differential equation, similar to the one obtained in the classical 1:1 reversibble resonance bifurcation, except that this system is non locally coupled with the complementary part, leading to a polynomial decay (instead of exponential) at infinity [1]. It is remarkable that the singularity corresponding to the merging of a pair of imaginary eigenvalues in the continuous spectrum at 0 , leads to a mode which satisfies the Benjamin-Ono ([9], [10], [11]) non local differential equation, giving in particular a polynomial decay of localized solutions at infinity [12], [13], [4], [2]. 


\section{EXAMPLE 1. A SINGLE FLUID LAYER WITH SURFACE TENSION AT THE FREE SURFACE}

We consider in this section the travelling waves occuring in a perfect infinitely deep fluid layer of constant density $\rho$, driven by gravity $g$, with surface tension $T$ at the free surface. The flow is assumed to be potential, and we write the equations in the frame moving at the velocity $c$ of the waves. In this example, the (dimensionless) bifurcation parameter is

$$
\varepsilon=\frac{g T}{\rho c^{4}}-\frac{1}{4}
$$

and we use the Levi-Civita variables $\alpha$ and $\beta$ defined in the complex plane by

$$
u-i v=e^{\beta-i \alpha}
$$

where $(u, v)$ are the components of the fluid velocity ( $\alpha$ is the slope of the stream lines while $\exp (\beta)$ is the modulus of the velocity). The coordinates are the velocity potential, denoted by $x$ and the stream function denoted by $y$. The transformation from the physical plane to the $(x, y)$ plane is a conformal map from the unknown domain of the flow, into the lower half plane, and the variable $U$ in equation (1) is defined by

$$
[U(x)](y)=\left(Z(x), \alpha_{0}(x), \alpha(x, y), \beta(x, y)\right)^{t},
$$

where $(1 / 4+\varepsilon)^{-1} Z(x)$ is the elevation of the free surface expressed in the new coordinates, and $\alpha_{0}(x)=\alpha(x, 0)$. The equation (1) may be written under the form

$$
\frac{d U}{d x}=F(\varepsilon, U)
$$

where

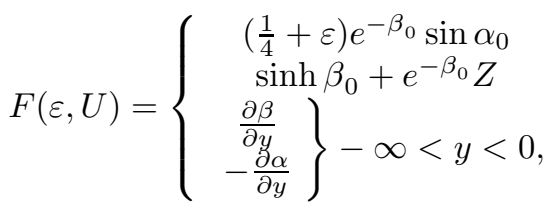

where we denote by $\beta_{0}$ the function $\beta(x, 0)$. Notice the Cauchy-Riemann system in the two last components of (1). The vector field $F(\varepsilon, U)(x)$ takes its values in a space $\mathbb{H}$ here defined by (various choices are possible)

$$
\mathbb{H}=\mathbb{R}^{2} \times\left\{C_{1}^{0}\left(\mathbb{R}^{-}\right)\right\}^{2}
$$

provided we take $U(x)$ in a smaller space $\mathbb{D}$ defined by

$$
\mathbb{D}=\mathbb{R}^{2} \times\left\{C_{1}^{1}\left(\mathbb{R}^{-}\right)\right\}^{2} \cap\left\{\alpha_{0}=\alpha(x, 0)\right\}
$$

where $C_{1}^{0}\left(\mathbb{R}^{-}\right)$is the space of continuous functions on $\mathbb{R}^{-}$, tending towards 0 as $1 / y$ at infinity, this being also true for their first derivatives for functions in $C_{1}^{1}\left(\mathbb{R}^{-}\right)$. The solution $U=0$ represents the rest state, with a flat free surface. The invariance of the system under the

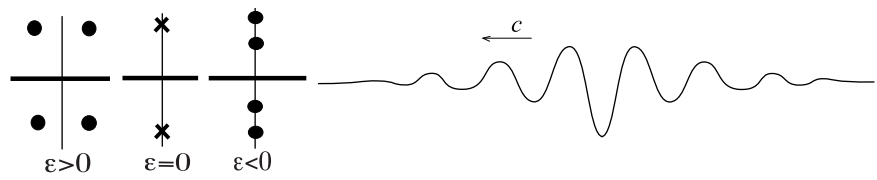

FIG. 1: Example 1 - Spectrum of $L_{\varepsilon}$ (left), and solitary wave for $\varepsilon>0$ (right)

symmetry $x \mapsto-x$, leads to a reversibility symmetry given by the linear operator $S$ such that

$$
S U=\left(Z,-\alpha_{0},-\alpha, \beta\right)^{t}
$$

which anticomutes with $F(\varepsilon, \cdot)$. The differential of $F(\varepsilon, \cdot)$ at the origin gives the linear operator $L_{\varepsilon}$ defined by

$$
L_{\varepsilon} U=\left(\left(\frac{1}{4}+\varepsilon\right) \alpha_{0}, \beta_{0}+Z, \frac{\partial \beta}{\partial y},-\frac{\partial \alpha}{\partial y}\right)^{t} .
$$

The eigenvalues $i k$ of this operator are such that the following dispersion equation is satisfied

$$
k^{2}-k(\operatorname{sgn}(\operatorname{Re} k))+\frac{1}{4}+\varepsilon=0
$$

which only gives at most 4 eigenvalues, and it can be easily shown (see [8]) that the whole real line constitutes the essential spectrum, with no real eigenvalue, and a non closed ranged for the operator $\sigma \mathbb{I}-L_{\varepsilon}$ when $\sigma$ is real, its closure being of codimension 1 . We see that for $\varepsilon=0$, we have a pair of double eigenvalues $\pm i / 2$, which split into two pairs of imaginary eigenvalues for $\varepsilon<0$, and two pairs of complex eigenvalues for $\varepsilon>0$ with

$$
i k_{ \pm}=\frac{i}{2} \pm \sqrt{\varepsilon}, \text { and }-i k_{ \pm},
$$

as shown on figure 1 (left side). The resolvent operator $\left(i k \mathbb{I}-L_{\varepsilon}\right)^{-1}$ for $k$ real, is such that

$$
\left\|\left(i k \mathbb{I}-L_{\varepsilon}\right)^{-1}\right\|_{\mathcal{L}(\mathbb{H})}<c /|k|,|k| \rightarrow \infty
$$

and near $k=0$ we have for $V=(a, b, f, g)^{t} \in \mathbb{H}$

$$
\left(i k \mathbb{I}-L_{\varepsilon}\right)^{-1} V=\eta_{\varepsilon}^{*}(V) \theta_{k}+S_{\varepsilon, k}(V)
$$

where the linear operator $S_{\varepsilon, k}$ is bounded for $V$ in a subspace dense in $\mathbb{H}$, and depends continuously on $k$ near 0 . The linear form $\eta_{\varepsilon}^{*}$ and the vector $\theta_{k} \in \mathbb{D}($ for $k \neq 0)$ are defined by

$$
\begin{aligned}
\eta_{\varepsilon}^{*}(V) & =(1 / 4+\varepsilon)^{-1} a+\int_{-\infty}^{0} g(y) d y \\
\theta_{k} & =\left(i \operatorname{sgn}(k),-1,-e^{|k| y},-i \operatorname{sgn}(k) e^{|k| y}\right)^{t} .
\end{aligned}
$$

The first term of the right hand side of (6) concentrates the singularity in $k=0$, due to the presence of the essential spectrum on the real axis. The range of $L_{\varepsilon}$ is such that $\eta_{\varepsilon}^{*}(V)=0$ where it is necessary that $g$ be integrable 


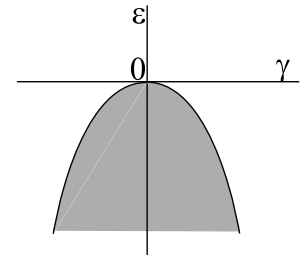

FIG. 2: Existence of "basic" periodic waves in the complement of the hatched region

on $\mathbb{R}^{-}$, which means that this range is not closed in $\mathbb{H}$ and the linear form $\eta_{\varepsilon}^{*}$ is continuous only on a subspace of $\mathbb{H}$.

Let us denote by $\zeta_{0}$ and $\zeta_{1}$ the eigenvector and generalized eigenvector such that

$$
\begin{aligned}
& \left(L_{0}-i / 2\right) \zeta_{0}=0, \quad S \zeta_{0}=\overline{\zeta_{0}} \\
& \left(L_{0}-i / 2\right) \zeta_{1}=\zeta_{0}, \quad S \zeta_{1}=-\overline{\zeta_{1}}
\end{aligned}
$$

then it is shown in [8] that there is a family of bifurcating periodic waves, with wave numbers $1 / 2+\gamma$ (close to $1 / 2)$, under the form of a power series convergent in $\mathbb{D}$, of the form

$$
\begin{aligned}
U(x)= & A e^{i s} \zeta_{0}+\bar{A} e^{-i s} \overline{\zeta_{0}}+i \gamma\left\{A e^{i s} \zeta_{1}-\bar{A} e^{-i s} \overline{\zeta_{1}}\right\} \\
& +\sum_{p+q \geq 1, r \geq 0, p+q+r \geq 3} A^{p} \bar{A}^{q} \gamma^{r} e^{i(p-q) s} U_{p q r} \\
\varepsilon= & \sum_{2 p+r \geq 2} \delta_{p r} \gamma^{r}|A|^{2 p}, \quad s=(1 / 2+\gamma) x
\end{aligned}
$$

where $|\gamma|$ and $|A|$ are small, $\delta_{02}=-1, \quad \delta_{10}>0$ $\left(=-q_{2}\right.$ defined below in (10)). When $A$ is real one has $S U(x)=U(-x)$, i.e. the solution is reversible. Moreover a phase shift on $A$ is equivalent to a shift of the $x$ origin. The method for proving this result mimics the proof of Lyapunov-Devaney theorem for finite-dimensional reversible systems (adaptation of the Lyapunov-Schmidt method). An easy adaptation in the infinite-dimensional case, as here, can be provided in using the estimate (5) at infinity. One additionnal difficulty here comes from the resonance at the origin which belongs to the essential spectrum. This difficulty is overcome thanks to the fact that for a periodic reversible $U(x)$ the range of the average of the nonlinear operator in (1) is included into the range of $L_{\varepsilon}$. This comes essentially from the fact that there is no nonlinear term in the two last components of the vector field (3). In the 4-dimensional reversible corresponding situation, we obtain additionnal families periodic with small wave number, and quasi-periodic solutions (see the corresponding proof in [14]). Their existence here is an open problem. The domain of existence of periodic waves (7) is described at figure 2 , in the region of the parameter plane $(\gamma, \varepsilon)$ complementary to the hatched region.
For the study of solitary waves, let us decompose

$$
\begin{aligned}
U & =V+W \\
V & =A \zeta_{0}+\bar{A} \overline{\zeta_{0}}+B \zeta_{1}+\bar{B} \overline{\zeta_{1}} \in E_{0}
\end{aligned}
$$

according to the natural decomposition comuting with $L_{0}$. In a way analogous to the finite depth case (see [7]), a normal form technique may be used (see [1]) to simplify up to the cubic order the equation projected on the 4dimensional subspace $E_{0}$ :

$$
V=X+\Phi_{0}(\varepsilon, X) \in E_{0}, \quad W=Y+\Phi_{1}(X)
$$

where $\Phi_{0}$ is cubic, and $\Phi_{1}$ quadratic in $X$. The system takes the new form

$$
\begin{aligned}
X_{x} & =L_{0} X+G(\varepsilon, X)+R_{0}(\varepsilon, X, Y) \\
Y_{x} & =\widetilde{L_{0}} Y+H(X)+R_{1}(\varepsilon, X, Y)
\end{aligned}
$$

where $\widetilde{L_{0}}$ is the restriction of $L_{0}$ to the codimension 4 subspace complementary to $E_{0}$, the rests satisfy the estimates

$$
\begin{aligned}
R_{0}= & O\left\{\| X \| \left(\varepsilon^{2}+\left|\varepsilon\|\mid X\|+\|X\|^{3}\right)+\right.\right. \\
& +\|Y\|(|\varepsilon|+\|X\|+\|Y\|)\} \\
R_{1}= & O\left\{\|X\|\left(\varepsilon+\|X\|^{2}\right)+\|Y\|(|\varepsilon|+\|X\|+\|Y\|)\right\},
\end{aligned}
$$

and $H$ is a quadratic function of $X$ only with resonant monomials, and the 4-dimensional vector field $L_{0} X+$ $G(\varepsilon, X)$ is the classical normal form for the reversible $1: 1$ resonance bifurcation, given by (denoting again by $A$ and $B$ the complex coordinates along $\zeta_{0}$ and $\left.\zeta_{1}\right)$

$$
\begin{aligned}
A_{x}= & (i / 2) A+B+i A\left\{p_{2}|A|^{2}+\left(i p_{3} / 2\right)(A \bar{B}-\bar{A} B)\right\} \\
B_{x}= & (i / 2) B+i B\left\{p_{2}|A|^{2}+\left(i p_{3} / 2\right)(A \bar{B}-\bar{A} B)\right\}+ \\
& +A\left\{\varepsilon+q_{2}|A|^{2}+\left(i q_{3} / 2\right)(A \bar{B}-\bar{A} B)\right\}
\end{aligned}
$$

where $q_{2}<0$. We see on the system above that $X$ and $Y$ are coupled in particular due to the rest $R_{0}$ which depends non locally on $Y$. Taking the Fourier transform of (9) allows to solve with respect to $Y$ by an implicit function argument, in a function space where $Y$ decays polynomially at infinity, provided that $X$ decays as $1 / x^{2}$. This resolution is possible here thanks to the estimate (5) on the resolvent and to the fact that for reversible solutions the discontinuous part of the resolvent (6) cancels when it is applied on the Fourier transform of the nonlinear terms. It then remains to solve (8), where $Y$ is replaced by its nonlocal expression in function of $X$. It is proved in [1] that this nonlocal ODE for $(A, B) \in \mathbb{C}^{2}$ has two reversible homoclinics, decaying as $1 / x^{2}$ at infinity (instead of an exponential decay as in the finite depth case) because of the polynomial decay of $R_{0}$. These solitary waves have, for finite $|x|$ a principal part identical to the one in the 4-dimensional case. The behavior at infinity is different, since the oscillations now decay polynomially, instead of exponentially (see figure 1 right side, for the shape of one of these solitary waves). 


\section{EXAMPLE 2. TWO SUPERPOSED LAYERS WITH NO INTERFACIAL TENSION AND STRONG SURFACE TENSION}

Let now consider a system of two superposed layers of perfect immiscible fluids (densities $\rho_{1}$ (upper layer) and $\rho_{2}$ (bottom layer)), assuming the flow to be potential in each layer and subject to gravity. We also assume that there is no surface tension at the interface and that there is indeed a strong enough surface tension at the free surface (for having the required property on the eigenvalues of the linearized operator $L_{\varepsilon}$ ). This case is treated in [2] and [3], as an example illustrating more general systems. The thickness at rest of the upper layer is $h$ while the bottom one has infinite thickness. We are interested in travelling waves of horizontal velocity $c$. The dimensionless parameters are $\rho=\rho_{1} / \rho_{2} \in(0,1)$ and

$$
\begin{array}{r}
\left.\left.\lambda=\frac{g h}{c^{2}} \text { (inverse of (Froude number }\right)^{2}\right), \\
b=\frac{T}{\rho_{1} h c^{2}} \text { (Weber number), }
\end{array}
$$

where $T$ is the surface tension at the free surface. We derive the equations in the frame moving with horizontal velocity $c$, and we use Levi-Civita variables and coordinates as in Example 1. The only difficulty is that we choose the $\underline{x}$ - coordinate as the velocity potential in fluid 2 (bottom layer), which introduces a factor function of $\underline{x}$ in the Cauchy-Riemann equations for the upper layer (fluid 1). The variable $\underline{U}$ in equation (1) is defined by

$$
\begin{array}{r}
{[\underline{U}(\underline{x})](y)=\left(\beta_{20}(\underline{x}), Z(\underline{x}), \alpha_{11}(\underline{x}), \alpha_{1}(\underline{x}, y), \beta_{1}(\underline{x}, y),\right.} \\
\left.\alpha_{2}(\underline{x}, \underline{y}), \beta_{2}(\underline{x}, \underline{y})\right)^{t},
\end{array}
$$

where $1+\frac{1}{2 \lambda}\left(1-e^{-2 \lambda Z(\underline{x})}\right)$ is the free surface, $\varepsilon$ is a distinguished parameter defined by $\varepsilon=1-\lambda(1-\rho)$ and, for example, $\alpha_{1 j} j=0,1$ means the trace of $\alpha_{1}$ in $y=$ 0,1 , and the same convention holds for $\beta_{i j}$. The right hand side of (1) is given by $F(\rho, \lambda, b ; \underline{U})=$

$$
\left\{\begin{array}{l}
-\lambda(1-\rho) e^{-3 \beta_{20}} \sin \alpha_{20}-\rho \frac{\partial \alpha_{1}}{\partial y}{ }_{\mid y=0} e^{3\left(\beta_{10}-\beta_{20}\right)}, \\
e^{2 \lambda Z-\beta_{11}+\beta_{10}-\beta_{20}} \sin \alpha_{11}, \\
\frac{e^{\beta_{11}}}{2 b}\left\{1-e^{-2\left(\lambda Z+\beta_{11}\right)}\right\} e^{\beta_{10}-\beta_{20}} \\
\left.\frac{\partial \beta_{1}}{\partial y} e^{\beta_{10}-\beta_{20}}\right\} y \in(0,1), \\
\left.-\frac{\partial \alpha_{1}}{\partial y} e^{\beta_{10}-\beta_{20}}\right\} y \in(-\infty, 0) . \\
\frac{\partial \beta_{2}}{\partial y} \\
\left.-\frac{\partial \alpha_{2}}{\partial \underline{y}}\right\} \underline{y} \in(-
\end{array}\right.
$$

The Galilean invariance of the physical problem induces a reflection symmetry (through the $y$ axis) of the system even in the moving frame. This invariance leads to the reversibility of system (1) under the reversibility symmetry $S$ defined by

$$
S \underline{U}=\left(\beta_{20}, Z,-\alpha_{11},-\alpha_{1}, \beta_{1},-\alpha_{2}, \beta_{2}\right)^{t},
$$

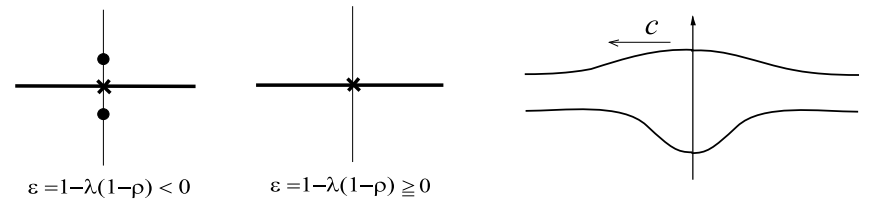

FIG. 3: Example 2 - Spectrum of $L_{\varepsilon}$ (left), solitary wave for $\varepsilon>0$ (right)

which anticommutes with the vector field $F(\rho, \lambda, b ;$.$) .$ The vector field (11) takes its values in $\mathbb{H}$ where

$$
\mathbb{H}=\mathbb{R}^{3} \times\left\{\mathcal{C}^{0}(0,1)\right\}^{2} \times\left\{\mathcal{C}_{1}^{0}\left(\mathbb{R}^{-}\right)\right\}^{2},
$$

provided that $\underline{U}(\underline{x})$ lies in $\mathbb{D}$ where

$$
\begin{aligned}
\mathbb{D}= & \mathbb{R}^{3} \times\left\{\mathcal{C}^{1}(0,1)\right\}^{2} \times\left\{\mathcal{C}_{1}^{1}\left(\mathbb{R}^{-}\right)\right\}^{2} \\
& \cap\left\{\alpha_{1}(0)=\alpha_{2}(0), \alpha_{11}=\alpha_{1}(1), \beta_{20}=\beta_{2}(0)\right\},
\end{aligned}
$$

and where we gave the definitions of spaces $\mathcal{C}_{1}^{0}\left(\mathbb{R}^{-}\right), \mathcal{C}_{1}^{1}\left(\mathbb{R}^{-}\right)$at Example 1. The system (1) has the same fundamental structure as (3), with the CauchyRiemann system in the lower half plane. This is the fact which implies the occurence of the essential spectrum of $L_{\varepsilon}$ on the entire real line. In this new example we have a one parameter family of solutions

$$
(0,-u / \lambda, 0,0, u, 0,0)^{t}, \quad u \in \mathbb{R},
$$

of the nonlinear system (1). This family of equilibria corresponds physically to a sliding with a non zero uniform velocity $u$ of the upper layer over the bottom one. This leads (in differentiating with respect to $u$ ) to the following eigenvector

$$
\xi_{0}=\left(0, \lambda^{-1}, 0,0,-1,0,0\right)^{t}
$$

belonging to the zero eigenvalue of the linear operator $L_{\varepsilon}$. In addition to the essential spectrum on the real line and the 0 eigenvalue, other eigenvalues $i k$ of $L_{\varepsilon}$ are isolated, with finite multiplicities, and given by the roots of the dispersion equation, defined for $\operatorname{Re} k>0$ by

$k\left(\rho b k^{2}+\lambda-k\right)=\tanh (k)\left\{\left(\lambda+b k^{2}\right)[\lambda(1-\rho)-k]+\rho k^{2}\right\}$,

which should be completed by the symmetric relationship (change $k$ into $-k$ ) for Re $k<0$. It can be shown on (12) that for $\varepsilon>0$ small enough, $b$ large enough and $1-\rho=$ $(q / b)^{1 / 3}$ with $0<q<4$, then 0 is the only real solution of the dispersion equation (12). This implies that for $\varepsilon<0$ there is only one pair of eigenvalues (close to 0 ) on the imaginary axis in addition to the 0 eigenvalue, and for $\varepsilon>0$ this pair disappears (see Figure 3, left side).

For studying the bifurcation occuring for $\varepsilon>0$ close to 0 , we need to make the following rescaling

$$
\begin{aligned}
\varepsilon \underline{x} & =x ; \varepsilon \underline{y}=y, y \in(-\infty, 0) ; y=y, y \in(0,1) \\
\underline{U} & =\varepsilon U,
\end{aligned}
$$


which leaves invariant the domain of the flow, and modifies the spectrum of the new linear operator $\mathcal{L}_{\varepsilon}$ in unfolding the singularity of the resolvent operator when $\varepsilon \rightarrow 0$. Equation (1) now reads

$$
\frac{d U}{d x}=\mathcal{L}_{\varepsilon} U+\mathcal{N}_{\varepsilon}(U)
$$

Since we intend to use the Fourier transform of (14) we need a careful study of the resolvent $\left(i k-\mathcal{L}_{\varepsilon}\right)^{-1}$ on the imaginary axis. When $|k| \rightarrow \infty$, the behavior is still given by (5), the difficulty here is the behavior near 0 . Indeed, for $V=\left(a_{1}, a_{2}, a_{3}, f_{1}, g_{1}, f_{2}, g_{2}\right) \in \mathbb{H}$, we have for $|k|<c / \varepsilon$

$$
\left(i k \mathbb{I}-\mathcal{L}_{\varepsilon}\right)^{-1} V=\frac{\xi_{\varepsilon, k}^{*}(V)}{i k \varepsilon \Delta} \xi_{0}+\frac{\eta_{\varepsilon, k}^{*}(V)}{\Delta} \theta_{k}+\varepsilon S_{\varepsilon, k}(V),
$$

where the first term on the right hand side is the component along the eigenvector $\xi_{0}$, and the rest which has the same form as (6), belongs to the kernel of the linear form $p_{0}^{*}$ satisfying $p_{0}^{*}\left(\xi_{0}\right)=1$, and defined by

$$
p_{0}^{*}(V)=g_{11} .
$$

In (15) the linear operator $\varepsilon S_{\varepsilon, k}(V)$ is bounded for $k \neq 0$, and continuous in $k=0$ when operating on a subspace dense in $\mathbb{H}$. We define the linear forms $\xi_{\varepsilon, k}^{*}$ and $\eta_{\varepsilon, k}^{*}$ uniformly bounded for $(\varepsilon, k)$ near 0 , when operating on a subspace dense in $\mathbb{H}$, and such that

$$
\begin{aligned}
\xi_{\varepsilon, k}^{*} & =\xi_{\varepsilon}^{*}+\varepsilon|k| \chi_{\varepsilon}^{*}+\zeta_{\varepsilon, k}^{*}, \\
\eta_{\varepsilon, k}^{*} & =\eta_{\varepsilon}^{*}+\beta_{\varepsilon, k}^{*},
\end{aligned}
$$

where $\zeta_{\varepsilon, k}^{*}$ and $\beta_{\varepsilon, k}^{*}$ are $O(k)$ and of class $\mathcal{C}^{1}$ near $k=0$. We also have

$$
\Delta(\varepsilon, k)=1+a|k|+O\left(\varepsilon k^{2}\right), a=\lambda^{-1}(\lambda-1)>0,
$$

which corresponds to the rescaling of the dispersion equation. The operator $\frac{\xi_{\varepsilon}^{*}(\cdot)}{\varepsilon} \xi_{0}$ is the projector on the eigenvector $\xi_{0}$, comuting with $\mathcal{L}_{\varepsilon}$, and the forms $\xi_{\varepsilon}^{*}, \eta_{\varepsilon}^{*}, \chi_{\varepsilon}^{*}$ are linked by

$$
a \xi_{\varepsilon}^{*}=\varepsilon \chi_{\varepsilon}^{*}-\eta_{\varepsilon}^{*}
$$

This implies that the closure of the range of $\mathcal{L}_{\varepsilon}$ is of codimension 2 (included in $\operatorname{ker} \xi_{\varepsilon}^{*} \cap \operatorname{ker} \eta_{\varepsilon}^{*}$ ). Here we have precisely

$$
\begin{aligned}
& \xi_{\varepsilon}^{*}(V)= a_{1}-\rho g_{10}+(1-\varepsilon) a_{2}+(1-\varepsilon) \int_{0}^{1} g_{1}(\tau) d \tau \\
& \eta_{\varepsilon}^{*}(V)= \lambda^{-1}(1-\lambda)\left(a_{1}-\rho g_{10}\right)-\rho a_{2} \\
&-\rho \int_{0}^{1} g_{1}(\tau) d \tau+\int_{-\infty}^{0} g_{2}(\tau) d \tau, \\
& \theta_{k}=\left(i \operatorname{sgn}(k), 0, \frac{-1}{\lambda-1}, 1-\frac{\lambda}{\lambda-1} y, 0, e^{|k| y}, i \operatorname{sgn}(k) e^{|k| y}\right)^{t},
\end{aligned}
$$

and we observe that

$$
\xi_{\varepsilon}^{*}\left(\theta_{k}\right)=i \operatorname{sgn}(k) .
$$

For the study of solutions tending towards 0 at infinity, we take the Fourier transform of (14), decomposed like $U$ as

$$
U=w \xi_{0}+\varepsilon Y, \quad p_{0}^{*}(Y)=0 .
$$

The equation for $Y$ may be solved with respect to $Y$ (where we look for a reversible $Y$ ) by an implicit function argument, as a nonlocal function of $w$, provided that $w$ is even and decays as $1 / x^{2}$ at infinity. The form of the resolvent at infinity (5) and near 0 (15) leads to a principal part for $Y$ of the form

$$
Y=a \mathcal{T} w+O(\varepsilon)
$$

where

$$
\widehat{\mathcal{T}(w)}=-i k \hat{w} \theta_{k}
$$

$\hat{w}$ representing the Fourier transform of $w$. This resolution with respect to $Y$ is the technical part of the mathematical study. The vector function $\mathcal{T} w$ lies in a space where, for a fixed $y$, the decay is in $1 / x^{2}$ at infinity. However the decays in $x$ and $y$ in the lower half plane are linked in such a way that the corresponding components are bounded by $c(1+|y|)\left(1+|x|^{2}+|y|^{2}\right)^{-1}$. It then remains a scalar equation for the function $w$, of the form

$$
w+a \mathcal{H}\left(w^{\prime}\right)-\frac{3}{2} w^{2}=\mathcal{B}_{\varepsilon}(w), \quad \mathcal{B}_{\varepsilon}=O(\varepsilon)
$$

where the Hilbert transform $\mathcal{H}(f)$ of a function $f$ is defined by

$$
\mathcal{H}(f)(x)=p \cdot v \cdot \int_{\mathbb{R}} \frac{f(s)}{x-s} d s .
$$

The left hand side of the above equation (21) is the Benjamin-Ono equation (see [9],[10],[11]), which is extensively studied in [15]. This equation plays the role of a "normal form", since it appears generically when, after moving a parameter, a pair of imaginary eigenvalues merge at 0 , disappearing in the continuous spectrum. In particular, the same study may be made if one replaces the upper free surface by a rigid plate (see [12], [13]) for the mathematical justification of the Benjamin-Ono equation, and see [2] for the use of the present method). The linear part of (21) may be found easily in applying the linear form $\xi_{\varepsilon}^{*}$ to equ. (14), in using (19), (18) and (20), and its Fourier transform corresponds to the multiplication by $i k \varepsilon \Delta(\varepsilon, k)$ (see $(16))$.

Using an implicit function argument it is then possible to prove the existence of an even solution of (21) of the form

$$
w(x)=u_{h}(x)+O(\varepsilon)
$$


which decays as $1 / x^{2}$, and where

$$
u_{h}(x)=\frac{4}{3\left(1+(x / a)^{2}\right)} .
$$

The shape of the corresponding solitary wave is shown on Figure 3 (right side). The above method may be used (see [2]) for proving the existence of similar solitary waves tending at infinity towards the non-zero steady solutions $\nu \xi_{0}$, for $|\nu|$ small, leading to solutions of (14) of the form

$$
\begin{aligned}
& U(x)=\left(\nu+u_{h}(x)\right) \xi_{0}+Y_{\varepsilon, \nu}(x), \\
& p_{0}^{*}\left(Y_{\varepsilon, \nu}\right)=0, \quad Y_{\varepsilon, \nu}=O(\varepsilon+|\nu|),
\end{aligned}
$$

where $Y_{\varepsilon, \nu}$ decays polynomially at infinity.

For the study of $a T$ - periodic waves, let us rescale $x$ as

$$
s=\frac{2 \pi}{a T} x
$$

then, we look for $2 \pi-$ periodic solutions $U(s)$ taking values in $\mathbb{D}$, of

$$
\frac{2 \pi}{a T} \frac{d U}{d s}=\mathcal{L}_{\varepsilon} U+\mathcal{N}_{\varepsilon}(U)
$$

Here again we split the space as

$$
U=u \xi_{0}+\varepsilon Y, \quad p_{0}^{*}(Y)=0,
$$

and it is shown in [3] that for reversible solutions, we can solve the equation for $Y$, with respect to $Y$ in function of $u$, thanks to the estimate (5) and thanks to the form of the resolvent near 0 , given in (15). We obtain $Y$ under the form

$$
Y=\mathcal{T}_{\natural, T}(u)+O(\varepsilon)
$$

where the $k^{t h}$ Fourier component of $\mathcal{T}_{\sharp, T}(u)$ is defined by

$$
\left\{\mathcal{T}_{\text {七,T }}(u)\right\}_{k}=-i \frac{2 \pi k}{T} u_{k} \theta_{\frac{2 \pi k}{a T}} .
$$

Now replacing $Y$ by its expression, the equation for $u$ takes the following form

$$
u+\frac{2 \pi}{T} \mathcal{H}_{\natural}\left(u^{\prime}\right)-\frac{3}{2} u^{2}=c+\mathcal{B}_{\natural, \varepsilon}(u),
$$

where $\mathcal{B}_{\natural, \varepsilon}=O(\varepsilon), c$ is an arbitrary constant, and $\mathcal{H}_{\natural}$ is the periodic Hilbert transform, such that $\mathcal{H}_{\natural}(\cos )=\sin$, $\mathcal{H}_{\natural}(\sin )=-\cos , \mathcal{H}_{\natural}($ const $)=0$. Let us introduce the 2-parameters family of periodic even functions

$$
\begin{array}{r}
u_{T, p}(x)=\frac{2 p v_{0}(p)}{3 T\left\{\cos ^{2}(x / 2)+\left(\frac{p v_{0}(p)}{2 \pi}\right)^{2} \sin ^{2}(x / 2)\right\}} \\
+\frac{1}{3}\left(1-\frac{p}{T}\right)
\end{array}
$$

where $v_{0}(p)$ is defined for $p \geq 2 \pi$, and solution of

$$
v_{0}^{2}-2 v_{0}+(2 \pi / p)^{2}=0 .
$$

Then $u_{T, p}$ is solution (see [15]) of (24), where $\mathcal{B}_{\natural, \varepsilon}=0$, and $c=\frac{1}{6}\left\{1-\left(\frac{p}{T}\right)^{2}\right\}$. It is proved in [3] that there is a family of "elliptic equilibria" with $u \sim u_{T, 2 \pi}$ constant, limits of periodic solutions where $p \rightarrow 2 \pi$. An implicit function argument allows to prove, for any fixed $p>$ $2 \pi$ except for some isolated values, the existence of a 2 parameters family of bifurcating periodic solutions of the form

$$
U=u_{T, p} \xi_{0}+O(\varepsilon)
$$

where we keep in mind that $c$ should stay small, i.e. $T$ should stay close to $p$ in the two parameters plane. This means that the period of the bifucating solutions of (14) runs from $2 \pi a$ until infinity (there is not yet any asymptotic result on the behavior of periodic solutions for periods tending towards infinity). We might remark that the results for the above Example 2, are very similar to the results obtained in the study of the bifurcations of a reversible 3 -dimensional vector field, where the linear operator has its 3 eigenvalues behaving in the neigborhood of 0 as here (see a complete study of such a case in [16], except that the homoclinics have a polynomial (instead of exponential) decay at infinity.

\section{EXAMPLE 3. TWO SUPERPOSED LAYERS WITH NO SURFACE TENSION}

The third Example is a limit case of Example 2, when $b=0$, i.e. with no surface tension at the free surface. Below, we give an idea of the method and results obtained in [4], and [5]. The system is again written under the form (1) with a vector field of the form (11), where $\underline{U}(\underline{x})$ has only 6 components, since for $b=0$, we have $\lambda Z+\beta_{11}=$ 0 , so the components $\left(Z, \alpha_{11}\right)$ are replaced by $\beta_{11}$. The linear operator $L_{\varepsilon}$ has again its essential spectrum filling the entire real axis and 0 is still an eigenvalue associated with the eigenvector

$$
\xi_{0}=(0,1,0,1,0,0)^{t}
$$

which corresponds to the existence of a one parameter family of flows where the upper layer slides with a constant velocity over the bottom layer. The eigenvalues $i k$ are isolated, with finite multiplicities, and satisfy the dispersion relation (12) where $b=0$. Then, we see that $i k= \pm i \lambda$ is a pair of imaginary eigenvalues, in addition to the pair of eigenvalues near 0 , occuring for $\varepsilon<0$. This last pair disappears for $\varepsilon>0$, and it still remains one pair of imaginary eigenvalues for $\varepsilon>0$ (see figure 4) (left side). Notice that if $b>0$ is small, we would obtain another pair of (large) imaginary eigenvalues. This would lead to a more complicated problem not yet studied at this moment.

In the present Example 3, the occurence of the additional pair $\pm i \lambda$ already leads to an oscillatory mode which interacts for $\varepsilon>0$ with the "critical" amplitude $w$ along $\xi_{0}$, we already had in Example 2. Indeed, here 


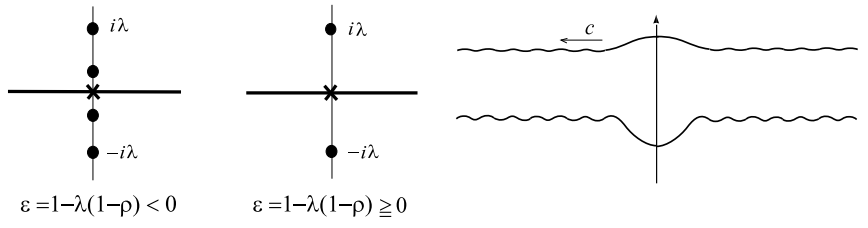

FIG. 4: Example 3 - Spectrum of $L_{\varepsilon}$ (left), and generalized solitary wave for $\varepsilon>0$ (right)

we need to combine both the techniques developed in Example 1 and in Example 2.

The first step is a rescaling (13) as in Example 2. This modifies the imaginary eigenvalues now being $\pm i \lambda / \varepsilon$ corresponding to eigenvectors $\zeta_{\varepsilon}$ and $\overline{\zeta_{\varepsilon}}$. The second step is the study of the resolvent operator $\left(i k-\mathcal{L}_{\varepsilon}\right)^{-1}$ which satisfies (5) at infinity, and which is of the form (15) near 0 , where in (16) the coefficient $a$ is $\rho$ (the density ratio). The third step consists in looking for periodic waves in using the same method as for obtaining the convergent series in powers of $\left(A_{0}, \overline{A_{0}}, u_{0}\right)$ (7) now of the form

$$
\begin{aligned}
U(x)= & p_{A_{0}, u_{0}, \varepsilon}(x)=A_{0} e^{i s} \zeta_{\varepsilon}+\overline{A_{0}} e^{-i s \overline{\zeta_{\varepsilon}}}+u_{0} \xi_{0}+ \\
& +\Phi_{\varepsilon}\left(A_{0} e^{i s}, \overline{A_{0}} e^{-i s}, u_{0}\right), \\
s= & \left(\frac{\lambda}{\varepsilon}+\gamma\right) x, \quad \gamma=\gamma\left(\left|A_{0}\right|^{2}, u_{0}, \varepsilon\right) .
\end{aligned}
$$

The fourth step is a change of variables, using the form of the family of periodic solutions

$$
U=A \zeta_{\varepsilon}+\overline{A \zeta_{\varepsilon}}+w \xi_{0}+\Phi_{\varepsilon}(A, \bar{A}, w)+Y
$$

where $Y$ lies in a complementary subspace of the 3dimensional space spanned by $\left(\zeta_{\varepsilon}, \overline{\zeta_{\varepsilon}}, \xi_{0}\right)$. The system satisfied by $(A, w, Y)$ takes the following form

$$
\begin{aligned}
\frac{d A}{d x} & =i A\left\{\frac{\lambda}{\varepsilon}+\gamma\left(|A|^{2}, w, \varepsilon\right)\right\}+R_{A}(A, \bar{A}, w, Y), \\
\frac{d w}{d x} & =p_{0}^{*} \mathcal{L}_{\varepsilon} Y+R_{w}(A, \bar{A}, w, Y), \\
\frac{d Y}{d x} & =\widetilde{\mathcal{L}_{\varepsilon}} Y+R_{Y}(A, \bar{A}, w, Y),
\end{aligned}
$$

which is reversible under the symmetry $S(A, \bar{A}, w, Y)=$ $(\bar{A}, A, w, S Y)$ and where $\widetilde{\mathcal{L}_{\varepsilon}}$ is the restriction of $\mathcal{L}_{\varepsilon}$ to the codimension-3 subspace of $Y$, and the rests $R_{A}, R_{w}, R_{Y}$ cancel for $Y=0$, which corresponds to the family of periodic solutions. Because of the coupling between the oscillatory mode and $w$, the idea is to look for solutions homoclinic to periodic solutions (generalized solitary waves). Then there is a new mathematical difficulty, due to the weak decay rate at infinity of the rest $R_{Y}$, with terms linear in $Y$. This necessitates the following treatment at step five: in [4] a normalization in presence of the continuous spectrum is provided with a change of variables depending analytically on $(A, \bar{A})$ which allows to change $R_{Y}(A, \bar{A}, w, Y)$ into $\Delta_{\varepsilon}(A, \bar{A}) Y+R_{Y}^{\prime}(A, \bar{A}, w, Y)$ where the new term $R_{Y}^{\prime}$ is decaying at infinity as a quadratic term in $(w, Y)$, and $\Delta_{\varepsilon}(A, \bar{A})$ is a linear operator depending analytically on $(A, \bar{A})$, taking its values in the kernel of $\eta_{\varepsilon}^{*}$ defined in (15). This last property allows to compensate the weaker decay at infinity of this term, with respect to $R_{Y}^{\prime}$, and it is possible at step six, to solve the last equation of system (25) with respect to $Y$, in a space of functions decaying algebraically at infinity, like in Examples 1 and 2, as a nonlocal function of $(A, \bar{A}, w)$ provided that $w$ decays as $1 / x^{2}$ and $\left.|| A|-| A\right|_{\infty} \mid$ decays as $1 / x$. Replacing $Y$ by its expression leads to a new system of the form

$$
\begin{aligned}
\frac{d A}{d x}-i A\left\{\frac{\lambda}{\varepsilon}+\gamma\left(|A|^{2}, w, \varepsilon\right)\right\} & =\mathcal{R}_{\varepsilon}(A, \bar{A}, w), \\
w+\rho \mathcal{H}\left(w^{\prime}\right)-\frac{3}{2} w^{2} & =\mathcal{B}_{\varepsilon}(A, \bar{A}, w)
\end{aligned}
$$

where the rests $\mathcal{R}_{\varepsilon}$ and $\mathcal{B}_{\varepsilon}$ are $O(\varepsilon)$. Here again we see Benjamin-Ono equation in the principal part of the second equation of (26). Now, the step seven consists in the study of system (26) for proving the existence of two families of generalized reversible solitary waves, of the form

$$
\begin{array}{r}
U_{A_{0}, \varepsilon}(x)=p_{A_{0}, 0, \varepsilon}\left\{x+\frac{\phi}{\pi} \arctan (x / \rho)\right\}+ \\
+u_{h}(x) \xi_{0}+O\left(\frac{\varepsilon+\left|A_{0}\right|}{1+|x|}\right)
\end{array}
$$

where there are two possible values of the phase shift $\phi$ at infinity, between the two sides of the limiting periodic wave for a given real amplitude $A_{0}$. The function $u_{h}$ is given in (23) where $a=\rho$, and the rest is small and decays as $1 /|x|$ at infinity. It is shown in [5], in using a precise analysis of the singularities in a strip containing the real axis in the complex $x$ - plane, that the minimal size of $A_{0}$ is $O\left(e^{-c / \varepsilon}\right)$, even though one can build a power series in $\varepsilon$ with all coefficients tending towards 0 at infinity (this is indeed a divergent series in general). It should be noticed that the results in Example 3 are less complete than in Example 2, and one conjectures the existence of other families of periodic and quasi-periodic solutions, and other generalized solitary waves asymptotic to periodic solutions where $u_{0} \neq 0$. Indeed, we think that the methods developed above (plus a lot of energy) might be the good tools for attacking such problems.
[1] G. Iooss, P. Kirrmann, Capillary Gravity Waves on the Free Surface of an Inviscid Fluid of Infinite Depth. Ex- istence of Solitary Waves. Arch. Rat. Mech. Anal. 136 
(1996) 1-19.

[2] M.Barrandon. Reversible bifurcation of homoclinic solutions in presence of an essential spectrum. To appear in J.Math. Fluid Mech.

[3] M.Barrandon. Family of Benjamin-Ono periodic solutions for a reversible bifurcation in presence of an essential spectrum. In preparation.

[4] G. Iooss, E. Lombardi, S.M. Sun, Gravity Traveling Waves for Two Superposed Fluid Layers of infinite depth : a new type of bifurcation, Phil. Trans. R. Soc. Lond. A 360 (2002), 2245-2336.

[5] E.Lombardi, G.Iooss. Gravity waves with polynomial decay to exponentially small ripples at infinity. Ann. I. Poincaré, 4 (2003) 669-704.

[6] K. Kirchgässner, Wave Solutions of Reversible Systems and Applications, J. Diff. Equ. 45 (1982), 113-127.

[7] F. Dias, G. Iooss, Water-Waves as a Spatial Dynamical System, Handbook of Mathematical Fluid Dynamics, vol II, S. Friedlander, D. Serre Eds, Elsevier (2003) 443-499.

[8] G. Iooss, Gravity and Capillary-Gravity Periodic Traveling Waves for Two Superposed Fluid Layers, one Being of Infinite Depth, J. Math. Fluid. Mech. 1 (1999) 24-61.
[9] T. B. Benjamin, Internal Waves of Permanent Form in Fluids of Great Depth, J. Fluid Mech. 29 (1967) 559-592.

[10] H. Ono, Algebraic solitary Waves in Stratified Fluids, J. Phys. Soc. Jpn, 39 (1975) 1082-1091.

[11] R.E.Davis, A.Acrivos. Solitary internal waves in deep water. J.Fluid Mech. 29 (1967) 593-607.

[12] C. Amick, On the Theory of Internal Waves of Permanent Form in Fluids of great Depth, Trans. Amer. Math. Soc. 364 (1994) 399-419.

[13] S.M. Sun, Existence of Solitary Internal Waves in a TwoLayer Fluid of Infinite Depth. Nonlinear Analysis 30 (1997) 5481-5490.

[14] G.Iooss, J.Los. Bifurcation of spatially quasi-periodic solutions in hydrodynamic stability problems. Nonlinearity, 3 (1990), 851-871.

[15] C. Amick, J. Toland, Uniqueness and Related Analytic Properties for the Benjamin-Ono Equation - a Nonlinear Neumann Problem in the Plane, Acta Math. 105 (1989) 1-49.

[16] G.Iooss. Travelling waves in the Fermi-Pasta-Ulam lattice. Nonlinearity, 13 (2000) 849-866. 